\title{
Photovoltaic Technology and Innovative Solar Cells
}

\author{
Nikolaos F. Voudoukis
}

\begin{abstract}
Photovoltaic technology has a range of applications nowadays. Organic solar cell research has developed in recent years. Common materials for organic solar cells are phthalocyanines. In this paper it is presented a discussion of the fundamentals of photovoltaic technology, photovoltaic effect, organic solar cells, phthalocyanines and Gallium Arsenide reconstructed surfaces. Also it is studied the behavior of lead pthalocyanine (PbPc) in substrates of Gallium Arsenide-GaAs (001) with reconstruction surface Arsenide (As) stoichiometric $\beta 2(2 \times 4)$. It is an analysis and study of innovative organic solar cells. The reason for this study is that PbPc is an organic molecule with very good optoelectronic properties, promising to be used in organic thin film solar cells or combination of organic and inorganic layers solar cells. In research is the behavior of phthalocyanines according to the surfaces of the substrates. The experimental photovoltaic characterization of a solar cell from lead phthalocyanine is presented. The efficiency of potential solar cells is estimated by measuring the photocurrent spectra. The understanding of the material and the samples combinations is important for potential applications and improvements for the use of solar cells.
\end{abstract}

Index Terms-Photovoltaic Technology; Organic Semiconductors; Organic Solar Cells; Phthalocyanines; Gallium Arsenide.

\section{INTRODUCTION}

Photovoltaic technology is the technology that generates electrical power from semiconductors when they are illuminated by light. Electricity is important to the humanity as it provides us with services such as electric light, radio, television, telecommunication, health services, clean water, cooling and warmth, electromechanical energy etc. [1]. Photovoltaic technology is of great importance as it uses solar energy for the production of electricity and/or heat (electrical plants/ electrical thermal plants). The study of photovoltaic technology aroused since the 1970s when oil fuel crisis happened. Then the consideration of alternative sources of energy was on demand and research for them increased. Another factor that has driven humanity towards the study of environmentally friendly forms of energy is the severe pollution of environment during this century such as the global warming. The humanity's environmental concern for global warming is declared by 191 countries who signed in December 1997 the Kyoto protocol in Japan, among those countries all members of European Union including Greece. Kyoto protocol is one the protocols of Rio Conference, held in 1992, that have been signed by countries universal for the protection of the environment and have global strength. Especially, Kyoto protocol declares the global interest to

Published on January 15, 2018.

N. F. Voudoukis is with Department of Electrical and Electronic Engineering Educators, School of Pedagogical and Technological Education (ASPETE), Athens, Greece (e-mail: nvoudoukis@ aspete.gr). reduce the Greenhouse gases: Carbon dioxide (CO2), Methane (CH4), Nitrous oxide (N2O), Hydrofluorocarbons (HFCs), Perfluorocarbons (PFCs) and Sulphur hexafluoride (SF6) [2]. The universal interest has turned to environmentally friendly forms of energy.

Even today, the 21 rst century, the one third of humanity does not have access to electricity and, therefore, to a large number of electricity-based services and commodities. Millions of people die every year from drinking polluted water, while others suffer from the lack of basic medical services. Illiteracy denies millions of people any possibility of gaining access to ideas for a better living. Electricity properly applied could help resolve many of these ailments of society and photovoltaic systems may be the best solution for rural electricity so as to ameliorate the life of the third world countries.

Solar energy is renewable, ecologically friendly and nonpollute energy supply contrary to fossil fuel that has produced the greenhouse effect, or nuclear energy. In addition, solar energy is more prosperous of the other green energy sources; wind, biomass and hydropower, geothermal as the technical knowledge are broader and it can provide us with sufficient energy.

Photovoltaic technology has a range of applications nowadays, from power supplies of satellites (since 1958) for telecommunication to remote sensing and navigation and applications in the roofs of houses or land for electrical supply. The benefits of the use of photovoltaic applications beyond the non-pollution of the environment are the zero operating cost, efficiency operation of 20-30 years, easy integration to facades and roofs of buildings, inclusion of them in constructions with parallel decrease of their building cost and possibility of expansion of the photovoltaic application. Another main advantage of the photovoltaic systems is that they can also be integrated in the current grid of electricity. The possibility of the creation of solar energy farms in desserts is also in consideration. Therefore, solar energy converted into electricity meets high expectations for the future in the field of applications which can also be the solution for rural and remote areas supplies [1].

The disadvantages of the solar cells are their considerably high cost of production (PVs cost more than the usual forms of energy e.g. coal) and the technical difficulties in fabrication of large area cells.

Countries that have turned to solar energy and have considerable photovoltaic power stations are USA, Germany, Canada, France, India, China, Italy, Spain, Portugal, Ukraine, Thailand, Czech Republic and Bulgaria. Greece although it has a lot of sun has not exploit this technology so much until now due to economic reasonsprivate households and lack of space. On the other hand, in Greece there are 6 big PV parks. First is the project of 10 MWp, which is located in Larissa by Selective Volt, a park 
in Beiotia (7.5 MWp) by Infoquest, Peloponnese (6 MWp) by EDF, two 5 MWp each one of Energa in Thebes, another of Positive Energy in Drama and a PV park in Eleutherios Venizelos airport with power $8 \mathrm{MWp}$.

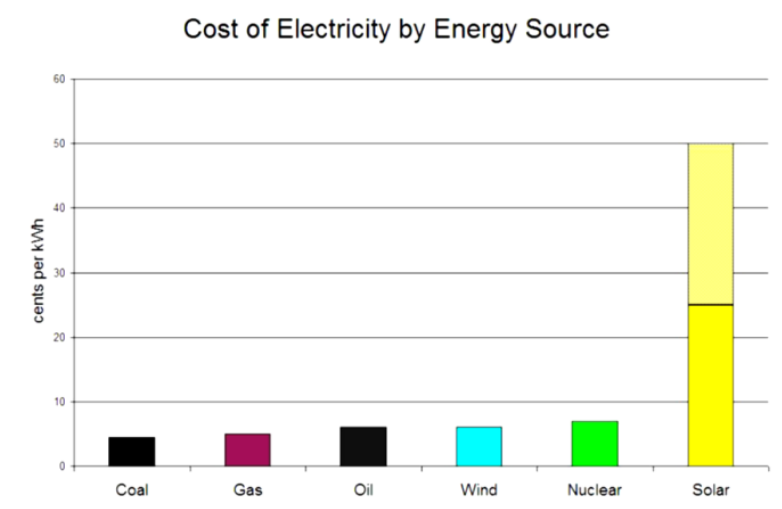

Fig.1. Cost of electricity by energy production source.

The above figure shows the cost of electricity by energy source. It is clearly depicted that solar energy is much more expensive than the other forms of energy supply. This is one major reason why it is not sufficiently used although it is beneficial from other sources. Note that solar energy bar reflects the fact that such energy can cost between $25 \phi$ and $50 \notin$ per $\mathrm{kWh}$, depending on the technology used and the estimated lifetimes of the cells [3].

\section{Photovoltaic EfFect}

Photovoltaic effect converts solar energy to electricity. The photovoltaic effect was initially reported by Edmund Becquerel in 1839, when he observed that the action of light on a silver coated platinum electrode immersed in electrolyte produced an electric current. The term "photovoltaic" comes from the Greek $\varphi \tilde{\omega} \varsigma$ (phōs) meaning "light", and from "volt", the unit of electro-motive force, the Volt, which in turn comes from the last name of the Italian physicist Alessandro Volta, inventor of the battery (electrochemical cell) in 19th century.

Solar radiation is consisted of photons, the particles of light each with quantized energy. The photons of the solar radiation with a sufficient energy are absorbed by proper semi conductive materials and their transfer of energy creates an exciton, an electron and a hole pair. The energy of photons must be higher than the energy gap of the semiconductor so as to be absorbed. In a photovoltaic device, however, there is some built-in asymmetry which pulls the excited electrons away before they can relax, and feeds them to an external circuit. The extra energy of the excited electrons generates a potential difference, or electromotive force. Electrons are driven to the negative (n) region of the semi conductive material and holes (a theoretical consumption which determines the lack of the electron) are driven to the positively (p) charged region of the semiconductor. Then the electrons pass to an external circuit with an application of a voltage through a selective contact where they produce electricity. Fig. 2 describes the physical basis of the photovoltaic effect in the solar cell. It is depicted a photovoltaic panel from a semiconductor with a p-type silicon layer and an n-type silicon layer.

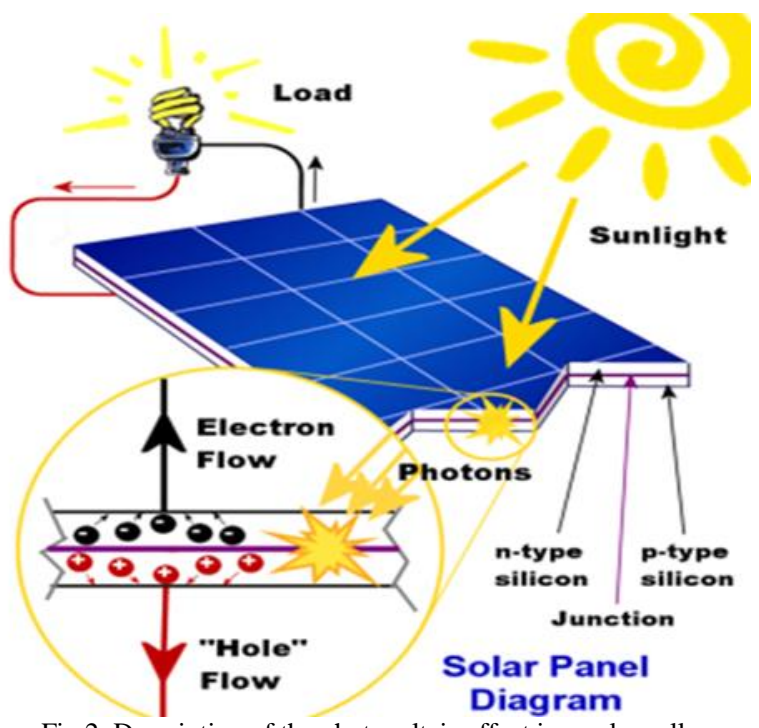

Fig.2. Description of the photovoltaic effect in a solar cell.

For p-type, silicon ( $\mathrm{Si}$ ) was doped positively with atoms called acceptors of electrons and negatively for n-type with atoms called donors of electrons. In between them is the $p-n$ junction where the photovoltaic effect occurs. Then the photons of equal or higher energy of the forbidden band, the band gap, give their energy to the atoms and each photon causes an electron to move from the valence band to the conduction band, leaving behind a hole also able to move around the material, thus give rise to an electron-hole pair, the charge carriers. When a load is positioned at the cell's terminals, electrons will pass through the external circuit, produce electricity, lose their energy and circulate back to the holes of the p-region [4].

\section{ORGANIC FILMS - ORGANIC SOLAR CELLS}

Photoelectric effect takes place when the incident light interacts with the electron in the medium with sufficient energy to unbound the electron from the medium (higher energy than the binding energy of the electron) [4].

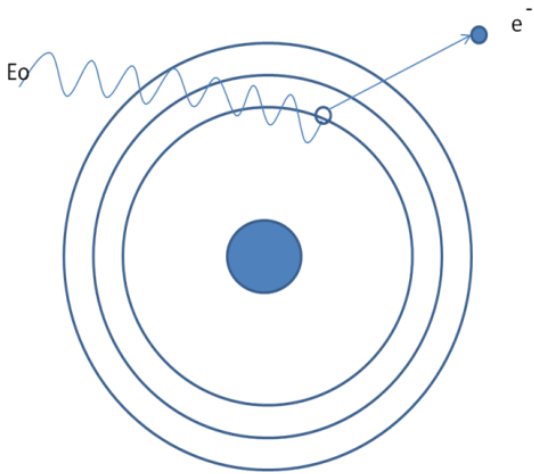

Fig.3. Photoelectric effect.

A solar cell is a device that converts the energy of the sun into electrical energy. This device is usually a semiconductor that has the capacity to absorb light and to deliver a portion of the energy of the absorbed photons to the carriers of the electrical current: electrons and holes. A semiconductor diode separates and collects the carriers and conducts the generated electrical current preferentially in a 
specific direction.

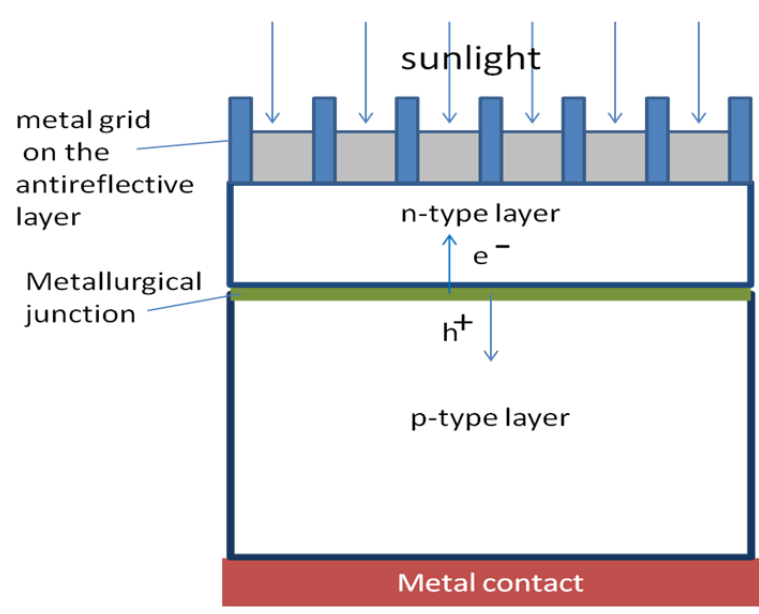

Fig.4. Simple conventional solar cell.

A schematic of a simple conventional solar cell is depicted in Fig. 3. Sunlight is incident from the top on the front of the solar cell. A metallic grid forms one of the electrical contacts of the diode and allows light to fall on the semiconductor in between the grid lines and thus be absorbed and converted into electrical energy. An antireflective layer between the grid lines increases the amount of light transmitted to the semiconductor. The metal grids are the electrodes that collect the electrons and lead them to the external circuit. They are more than two so that the collection of electrons is sufficient and they do not recombine with holes [1]. A solar cell with the following layers: metal contact at the bottom, $\mathrm{p}$-doped region, $\mathrm{n}$-doped region, an antireflective layer and metal grids. Between the p-n junction we have the creation of an electron-hole pair with the absorption of the sunlight.

Scientific and industrial laboratories research further for new material (organic compounds containing carbon - of the 2000 compounds known, approximately $90 \%$ are organic) for photovoltaic technology from 1950's. The photovoltaic effect was first observed in an organic material in the 1960s after it was discovered that some common dyes had semiconducting properties. The breakthrough in the field came in 1986 due to Tang's patent of creating a heterojunction in solar cells. In 1991 Gratzel created the first dye-sensitized solar cell with an efficiency of $11 \%$, the greatest reported until now in organic solar cells. Common materials for organic solar cells are phthalocyanines (especially metal phthalocyanines - p type materials), porphyrins, chlorins and closely to those tree macrocycles. The first requirement is the harvesting of a significant fraction of the solar spectrum. Almost half of the total energy of sunlight appears at wavelengths below $700 \mathrm{~nm}$, a region typically covered well by the porphyrin and phthalocyanine families. Porphyrins and related derivatives having extended conjugation are a common approach to extending the range of the absorption spectrum into the near infrared.

A problem that is raised in organic materials is to get the charges (electron-hole) that have been created through the electrodes. Electron and hole mobilities are low for most organic compounds. Hence conductive polymers are of particular interest. Polythiophenes and poly (phenylenevinylenes) are two of them. In addition, porphyrins and their derivatives organized as liquid crystals or as conductive nanostructured polymers have been studied as improved charge carrier materials for solar cells.

Organic films deposited on semiconductor surfaces have yield interest due to their semi-conductive properties which make them candidates for optoelectronic and electronic devices such as organic field effect transistors (OFETs), organic light emitting diodes (OLEDs), chemical sensors and organic solar cells. Another important factor of the heterostructures of organic films on semi-conductive materials is their modification in terms of their optoelectronic and electronic properties by changing the assembly of the molecules or the semi-conductive surfaces they are absorbed by.

Organic solar cell research has developed during the past 30 years, but especially in the last decade it has attracted scientific and economic interest triggered by a rapid increase in power conversion efficiencies. This was achieved by the introduction of new materials, improved materials engineering, and more sophisticated device structures. Today, solar power conversion efficiencies in excess of $3 \%$ have been accomplished with several device concepts [5], [6]. Laboratories efficiencies reach 6\%-7\%. Generally, efficiencies of these thin-film organic devices have not yet reached those of their inorganic counterparts $(\eta \approx 10-20 \%)$ but the perspective of cheap production (employing, e.g., roll-to-roll processes) drives the development of organic photovoltaic devices further in a dynamic way. The organic solar cells in the market have the schematic of the Fig. 4. The materials vary from having the central layer organic or the central three layers organic and the characterized respectively to the materials they are made of.

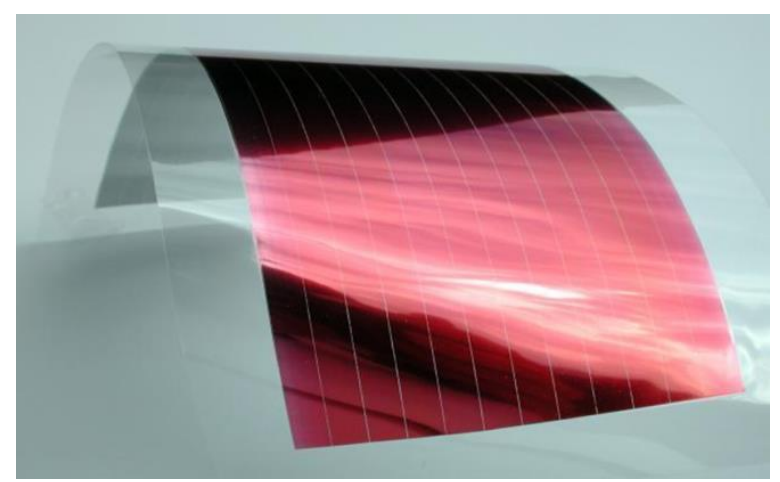

Fig. 5. Thin film organic solar cell from Fraunhofer ISE.

One of the principle difficulties when trying to fabricate an organic solar cell is the instability of most cell materials in ambient conditions. Most of the cells presented in the literature are fabricated and characterized without ever being exposed to air. They also have very short lifetimes, making them useless in any real application without some type of encapsulation that would also add to the cost and difficulty of manufacture. Those disadvantages need more research to solve them [5], [7].

The first report was made by Tang in 1986, as it is mentioned above, and it is an organic solar cell composed of $\mathrm{Cu}$-phthalocyanine and a perylene derivative. Since then the field expanded rapidly and now includes a wide variety of "small" molecule solar cells containing phthalocyanines or porphyrins as dyes, conjugated polymers as the p-type donor phase, and C60 or perylene derivatives as the acceptor phase. 
Research on organic solar cells - OPVs (organic photovoltaics) generally focuses either on solution process able organic semiconducting molecules/polymers or on vacuum-deposited small-molecular materials. As mentioned before, phthalocyanine is a p-type, hole conducting material that works as electron donor, whereas perylene and its derivatives show an n-type, electron conducting behavior and serve as electron-acceptor material.

In general, organic semiconductors can be regarded as "intrinsic wide band gap semiconductors" (band gaps above $1.4 \mathrm{eV}$ ) down to "insulators" (band gaps above $3 \mathrm{eV}$ ) with a negligibly low intrinsic charge carrier density at room temperature in the dark. Chemical, photochemical, or electrochemical doping is used to introduce extrinsic charge carriers into organic semiconductors. For example, photoinduced electron transfer from a donor to an acceptor-type organic semiconductor film introduces free charge carriers (positive charge carriers on the donor layer, i.e., p-type, and negative charge carriers on the acceptor layer, i.e., n-type). Donor-acceptor-type bi-layer devices can thus work like classical p-n junctions. Chemical doping of a semiconductor matrix by introducing small concentrations of reagents (dopants) has also been reported [8].

\section{PHTHALOCYANINES}

The family of phthalocyanines (Pcs) represents one of the most promising candidates for organic thin films as these systems offer excellent film growth properties as well as possessing advantageous optoelectronic properties and chemical stability, low cost, flexibility in functional group substitution and high tendency of self-organization [9]. Pcs are particularly prominent in molecular solar cells. They have been used as important dyes and pigments since their synthesis at the beginning of the last century. In recent years, phthalocyanine derivatives have been used as charge carriers in photocopiers and laser printers and materials for optical storage. The semiconducting properties of single phthalocyanines have been studied since the 1960's and in recent years there has been a renewed interest in small molecular films, their growth and electrical properties. Phthalocyanines are also used to Dye- sensitized solar cells. In addition, Pc's have similar structural relationship to chlorophyll, which performs the sunlight harvesting for the ultimate solar energy conversion process, photosynthesis, gives further encouragement to their use in solar cells.

Phthalocyanine $(\mathrm{Pc})$ is a symmetrical 18 p-electron aromatic macrocycle of four iminoisondole units, closely related to the naturally occuring porphyrins. Like the porphyrins, the Pc macrocycle can play host to over seventy different metal ions in its central cavity and the choice of central metal cation can strongly influence its physical properties. Especially, the central cavity of phthalocyanines is known to be capable of accomodating 63 different elemental ions, including hydrogens (metal-free phthalocyanine, H2-PC). A phthalocyanine containing one or two metal ions is called a metal phthalocyanine (M-PC). In the last decade, as a result of their high electron transfer abilities, M-PCs have been utilized in many fields such as molecular electronics, optoelectronics and photonics. More recently they have been employed in several 'hi-tech' applications such as the photoconducting material in laser printers and the light absorbing layer in recordable CDs. They are also used as photosensitisers in laser cancer therapy, as nonlinear optical materials and as industrial catalysts. In photodynamic therapy of cancer, they have been used as they have strong absorption in far red light at the $(650 \mathrm{~nm}$ to $800 \mathrm{~nm})$. The name was conceived by the researcher Linstead as a combination of the prefix phthal, originally from the Greek naphtha (rock oil) to emphasize the association with its various phthalic-acid- derived precursors and the Greek cyanine, blue. Their use as industrial colorants started at 1935 (manufacture of copper Pc) and Pcs are not found in nature. Another great application of phthalocyanines is in the experiments of molecular physics due to their stability. Therefore, for many new experimental techniques they have bridged the gap between crystalline inorganic materials (e.g. metals and ionic crystals) originally used to develop the technique and their application to molecular materials.

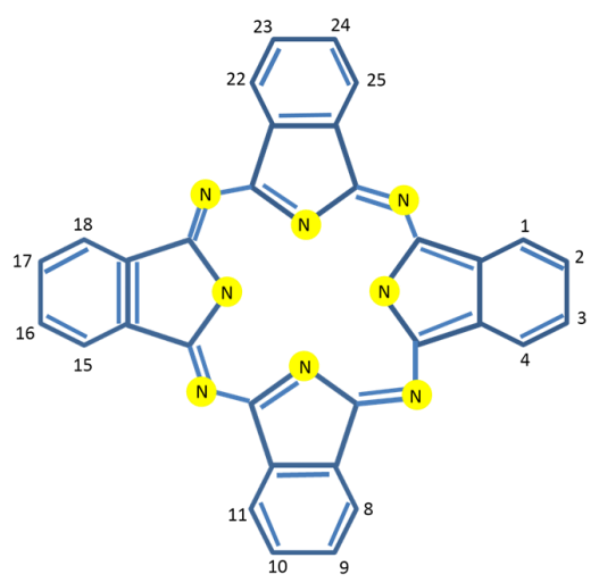

Fig. 6. The structure of phthalocyanine.

To name some mettalophthalocyanines: copper Pc $(\mathrm{CuPc})$, vanadyl Pc (VOPc), magnesium Pc (MgPc), zinc Pc $(\mathrm{ZnPc})$.

The structure of phthalocyanine is represented (Fig. 5) with each of the possible sites of substitution at the benzopositions. We can see sixteen possible sites for macrocycle substitution. The 2,3,9,10,16,17,23,24 carbon atoms are termed the (p) peripheral sites and the 1,4,8,11,15,18,22,25 are denoted the non-peripheral sites (np).

\section{GAllium ARSEnide ReCONSTRUCted Surfaces}

Gallium Arsenide (GaAs) is a semi conductive material, III/V respectively of the periodic table and it is often used for epitaxial growth of semiconductors. It is very dark red in appearance; it has a band gap of $1,424 \mathrm{eV}$ in $300 \mathrm{~K}$, its crystal structure is zinc blende and it has a direct band gap which results in infrared emission $\sim 870 \mathrm{~nm}$ [10]. Furthermore, it is advisable to mention that Arsenide is a toxic material and possibly carcinogen.

The zinc blende or Diamond, or Sphalerite lattice consist of a face centered cubic (fcc) Bravais point lattice which contains two different atoms per lattice point. The distance between the two atoms equals one quarter of the body diagonal of the cube. 
Generally, the zinc blende and the diamond structures are important because the majority of the semiconductors belong to this class. To mention some of them $\mathrm{Si}$, Ge have diamond structure and as mentioned before GaAs and $\mathrm{ZnSe}$ zinc blende.

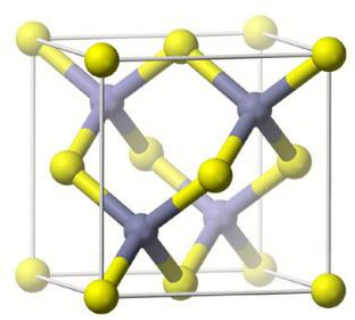

Fig. 7. Zinc blende structure. Yellow is Ga and purple As.

In the experiment described it is used GaAs (001). Those are the directions of GaAs crystalline material. Nearly all semiconductor materials are considered cubic crystals because they all can be formed by interpenetrating several simple cubic lattices. Therefore (001) are the direction indices- Miller's indices of GaAs we use.
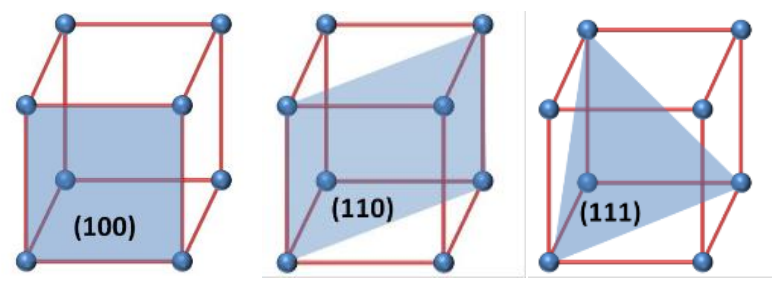

Fig. 8. Most important planes of a cubic cell (100), (110) and (111)

The GaAs surfaces reconstruction of (100) it is studied used experimentally in the hybrid cells are $\beta 2(2 \times 4)$ which is moderately as rich - stoichiometric surface.

There are depicted the top views of GaAs (001) reconstruction surfaces which were used in the experiment as substrates. Empty and filled circles represent $\mathrm{Ga}$ and as respectively. Larger circles indicate the position of the atoms in the uppermost two atomic layers.

There are another 4 main reconstruction surfaces of this GaAs (001), those are:
- $(2 \times 6)$
As rich
- $\alpha 2(2 \times 4) \quad$ moderately Ga rich
- $\zeta(4 \times 2) \quad$ Ga rich
- $\quad$ mixed dimmer.

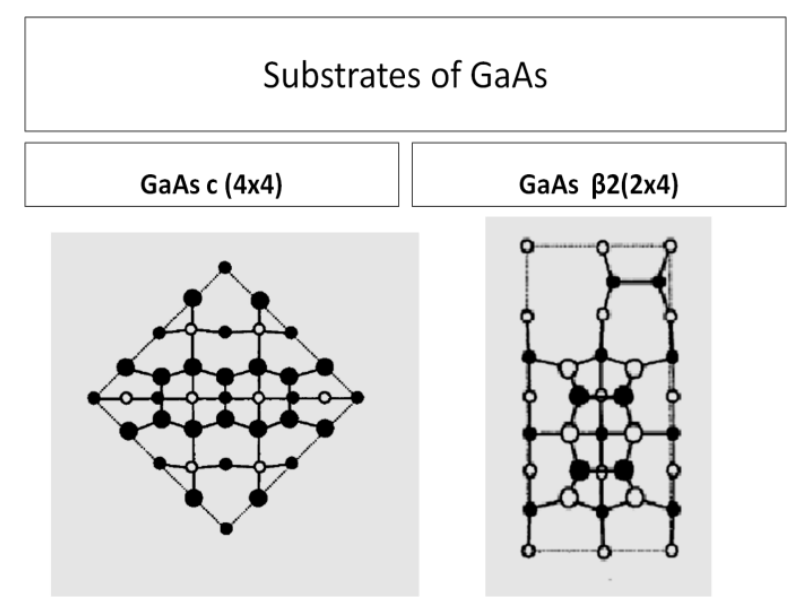

Fig. 9. Top views of GaAs (001) $c(4 \times 4)$ and $\beta 2(2 \times 4)$.

\section{DESCRIPTION OF AN EXPERIMENT}

The experimental photovoltaic characterization of a solar cell from lead phthalocyanine ultra-thin film of $20 \mathrm{~nm}$ deposited on a reconstruction surface of $\mathrm{PbPc} / \mathrm{GaAs}$ (001)$\beta 2(2 \times 4)-$ is presented. The efficiency of potential solar cells is estimated by measuring the photocurrent spectra. The photocurrent spectra is measured using an optical set-up made up of a xenon lamp equipped with a monochromator, focusing and collecting optics, a reflecting chopper and lock-in electronics. A Xenon lamp is used because the spectrum of this lamp is very similar to that of the sun. Therefore, the sun can be simulated to our solar cell. The Xe lamp is made of a glass holder with two electrodes inside.

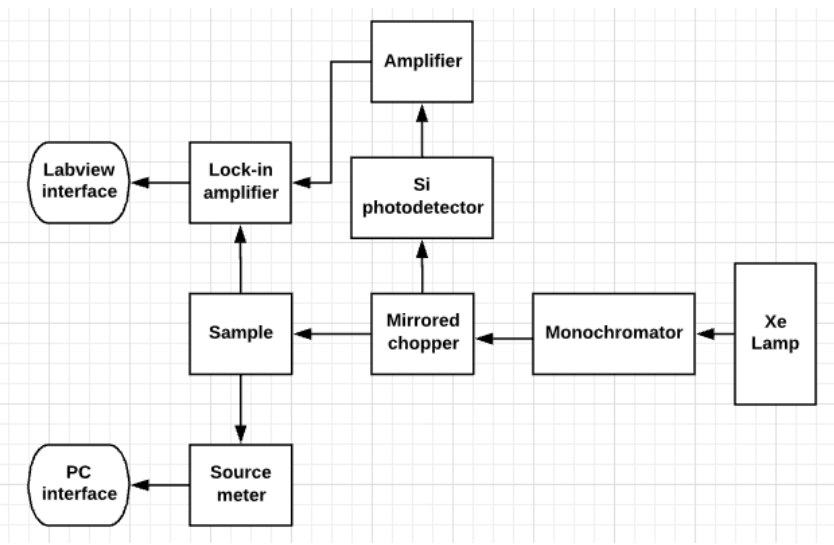

Fig. 10. The set-up of the experiment.

In the Xe gas, the atoms of the gas are excited by electrical discharge which is created by the presence of a high voltage between the electrons, in the electronics of the Xe lamp. The light passes through the monochromator selecting the entire wavelength range. The light is monochromatized and then the lens focuses the spot light once to the sample and the other to the photodiode through the reflective chopper. The reflective chopper made from aluminum allows the light one time to go to the silicon photodiode and from the photodiode to lock-in electronics to measure the incident power and the other time to reach the sample so the sample absorbs the light and produces photovoltaic power. Those two powers are compared and they give the External Quantum Efficiency (EQE) of the sample. The equation of $\mathrm{EQE}$ is:

$E Q E(\lambda)=\frac{h c I(\lambda)}{e \lambda P(\lambda)} 100$

The sample holder is able to move the sample in three dimensions $(\mathrm{x}, \mathrm{y}, \mathrm{z})$ so as the sample is aligned with the light. The photodiode is a photodetector that converts light into voltage in the experiment. Generally, a photodiode can convert light into current or voltage depending upon the operation. A photodiode is a p-n junction. This photodiode was made from silicon ( $\mathrm{Si}$ ) with an electromagnetic spectrum wavelength range from $190 \mathrm{~nm}$ to $1100 \mathrm{~nm}$.

The current is too small and it is used an amplifier to enlarge it so that it can be measured by the lock-in electronics. The lock-in electronics delete the background which comes from the light of the room and the electronics. The source meter measures the I-V characteristic graphs. 
The low noise amplifier is needed in the experiment because of the low signal in the sample (micro amperes- $\mu \mathrm{A}$ ) which is needed to be amplified (when samples give higher currents the low noise amplifier is not in use).

This instrumentation measures the I-V curves and the external quantum efficiency of a solar cell. With EQE we know the optical response of the sample for every wavelength while with the I-V curves we know the parameters of the solar cell:

- $\quad$ Short circuit current, $\mathrm{I}_{\mathrm{sc}}$

- Open circuit voltage, $\mathrm{V}_{\mathrm{oc}}$

- Maximum power point, MPP

Metallic electrodes of silver (Ag) in solution (silver and solvent acetone which evaporated) are dropped on the sample $\mathrm{PbPc} / \mathrm{GaAs} \beta 2(2 \times 4)$. Silver is used because it has good conductivity so that the photocurrent is more intense.

Two different sample holders can be used to create different current flow for the samples. The samples holders create the two different configurations: the top - down configuration and the planar configuration. Top - down configuration is when the current collected from the bottom and the top electrodes at the same time while in planar configuration the current is collected from the top electrodes only. In the figures the arrows show the direction of the current flow. The reason to study both configurations is to see which configuration has better signal to optimize the way to harvest solar energy.

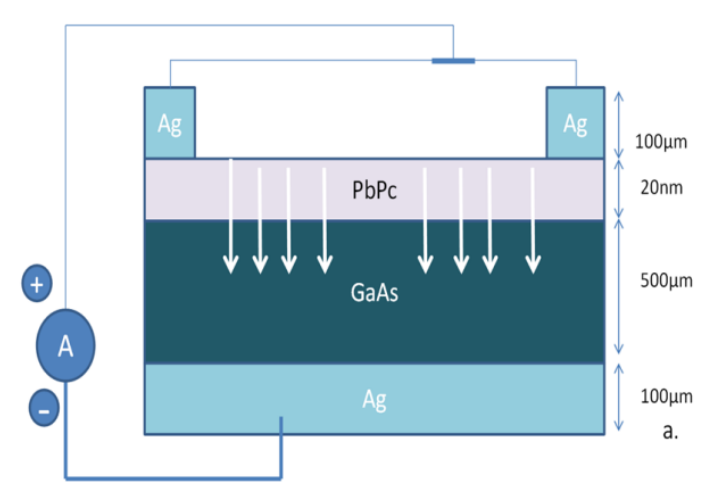

Fig. 11. Top-down configuration.

The sample is depicted and we can see the connection between the front $\mathrm{Ag}$ parts with the bottom $\mathrm{Ag}$. The front parts of Ag connected create the positive electrode and the bottom the negative electrode. The arrows depicted the current flow (top-down configuration) which is created by the first sample holder. GaAs is n-type (donor) and $\mathrm{PbPc}$ is p-type (acceptor).

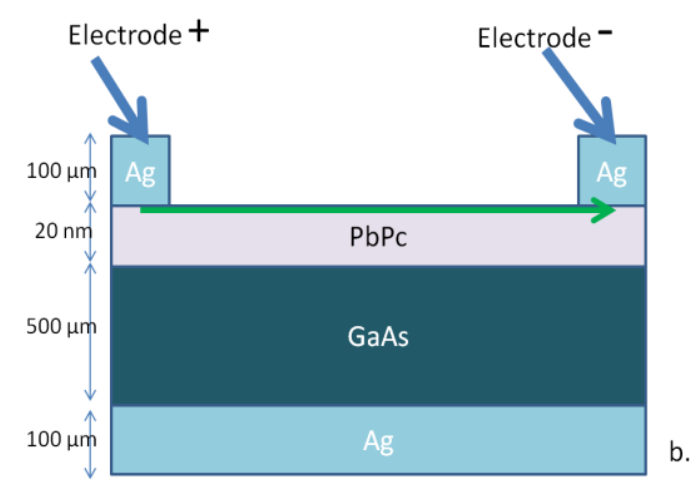

Fig. 12. Planar configuration.

At the planar configuration the direction of current flow through the layer of $\mathrm{PbPc}$ is depicted by the arrows. PbPc is the active layer where the creation of the exciton happens and this layer includes the electrons that pass through the negative electrode to the external circuit and are collected back to recombine with the holes through the positive electrode.

The photocurrent density $I(\lambda)$, is measured under illumination as a function of the incident photon wavelength, $\lambda$. The incident photons to current efficiency is defined as the fraction of the incident photons $\mathrm{N}_{\mathrm{ph}}$, converted into photocurrent, i.e. the number of the generated electron-hole pairs, multiplied by the electronic charge. The number of the incident photons is then evaluated in terms of the power density $P(\lambda)$ of the Xe lamp, since $N_{p h}=\frac{\lambda P(\lambda)}{h c}$. Therefore, it results to Incident Power Cell EfficiencyIPCE:

$\operatorname{IPCE}(\%)=\frac{\text { electrons }}{\text { photons }}=\frac{100 \mathrm{hc} \mathrm{I}(\lambda)}{\lambda \mathrm{P}(\lambda)}$

Where $I(\lambda)$ is measured by modulating the light by an optical chopper and recovering the amplified current signal (converted to voltage) by a lock-in amplifier locked on the chopper frequency. The lamp power $P(\lambda)$ is measured simultaneously in a similar way by a calibrated $\mathrm{Si}$ photodiode. Finally, the current is recorded by positioning the light spot at variable distance from the metallic contact electrode, from which the signal is measured and to which the bias can be applied both in dark and under illumination and we obtained the current-voltage curves. Generally, solar cells are measured in Standard Test Conditions (STC) which is a temperature of $25^{\circ} \mathrm{C}$ and an irradiance of $1000 \mathrm{~W} / \mathrm{m}^{2}$ with an air mass 1.5 (AM1.5) spectrum. These conditions correspond to a clear day with sunlight incident upon a sunfacing $37^{\circ}$-tilted surface with the sun at an angle of $41.81^{\circ}$ above the horizon.

Gallium Arsenide (GaAs) is measured in order to compare the photoresponce of the $\mathrm{PbPc} / \mathrm{GaAs}$ with that of uncovered GaAs substrate. The substrate forms a Schottky junction due to the contact of Ag and GaAs. In solar cells we ought to have at the electrodes Ohmic and not Schottky junction between the layers of the solar cells so as the photocurrent should flow without resistances. Graphs of $\mathrm{PbPc} / \mathrm{GaAs} \beta 2(2 \times 4)$ are used to compare it from the bear substrate- GaAs and thus estimate how efficient this solar 
cell is and how much PbPc layers have ameliorate their efficiency.

From power versus voltage curve of $\mathrm{PbPc} / \mathrm{GaAs} \beta 2(2 \times 4)$ one can (graphically) determine the current and voltage of the Maximum Power Point. Short circuit current $\left(\mathrm{I}_{\mathrm{sc}}\right)$ and open circuit voltage $\left(\mathrm{V}_{\mathrm{oc}}\right)$ are the other two parameters that describe a solar cell.

There is a double diode behavior (Fig. 13). This is due to the presence of another junction beyond the $\mathrm{PbPc}-\mathrm{GaAs}$ Schottky junction which we want to measure. The other Schottky junction created is between GaAs and Ag. Here, a not Ohmic junction as it should be, but another Schottky junction is formed (especially in top-down configuration). Generally, solar cells have an Ohmic junction at all their electrodes.

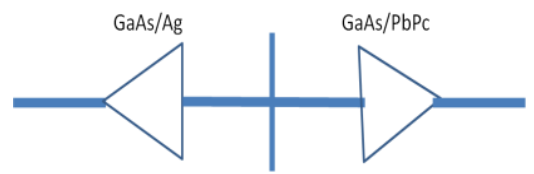

Fig. 13. Double diode behavior. The phenomenon of a double diode behavior is due to two continuous and different junctions.

\section{CONCLUSION}

In research is the behavior of phthalocyanines according to the surfaces of the substrates. Lead pthalocyanine $(\mathrm{PbPc})$ is an organic molecule with very good optoelectronic properties, promising to be used in organic thin film solar cells or combination of organic and inorganic layers solar cells. PbPc belong to the category of metal phthalocyanines (which are also organic materials) and they change their molecular ranking when they are deposit on different substrates by taking the orientation of the surface reconstruction of the substrate, therefore they change their absorption quality. The experimental research on those combinations and the results can show to the scientific and industrial society which sample is efficient and its behavior on different surface reconstructions of GaAs (001). This understanding of the material and the samples combinations is important for potential applications and improvements for their use as molecular solar cells as well as their use as thin film interlayer to improve electronic and optoelectronic devices.

In order to improve the solar cells, we have to:

a. Create an Ohmic contact at the bottom electrode and top electrode. This can be done with the usage of an intermediate material between $\mathrm{GaAs}$ and $\mathrm{Ag}$ with a work function similar to the work function of GaAs. Solar cells in the market have Ohmic junctions between their electrodes and the semi conductive material. The phenomenon of the double diode behavior is due to that.

b. Reduce the series resistance. The reduction of series resistance with increase the power generated by the solar cell. For this we can put more electrodes (in a grid-like structure) at the front so as to collect more electrons. In this way the path of an electron collected and transferred to the external circuit is smaller, therefore, the resistance too. Generally, the thickness of the phthalocyanine layer is ultrathin films of about $25 \mathrm{~nm}-300 \mathrm{~nm}$. Experiments can be done with thick layers for the reduction of the resistance.

It is important to mention that many losses in the efficiency can be counted. These are: a. Reflectance losses which are accounted for by the quantum efficiency value, as they affect "external quantum efficiency." b. Recombination losses are accounted for by the quantum efficiency, volatile organic compounds (VOC) ratio, and fill factor values. c. Resistive losses are predominantly accounted for by the fill factor value, but also contribute to the quantum efficiency and VOC ratio values.

Generally, in a solar cell like the sample from $\mathrm{PbPc} / \mathrm{GaAs}$ (001) electrons from $\mathrm{PbPc}$ should be collected from the top silver electrodes and electrons from GaAs from the bottom silver layer. If the top electrodes are in liquid phase, they may penetrate the layer of $\mathrm{PbPc}$ creating a direct contact with the GaAs, therefore result to a short circuit. This decreases the performance of the sample by reducing in the Voltage Open Circuit. A way to avoid the short circuit is to put an insulating layer between silver and GaAs.

\section{REFERENCES}

[1] S. Hedegus, A. Luque, Handbook of Photovoltaic Science and Engineering, Wiley Editorial Office, 2003.

[2] H. Hoppe and N. S. Sariciftci, Organic solar cells: An overview, Journal of Materials Research, volume 19, pg. 1924-1945, 2004.

[3] P. Wurfel, Physics of solar cells, second edition, Wiley, 2009.

[4] N.B. McKeown, Phthalocyanine Materials, Chemistry of Solid state, edition 6th, 1998.

[5] C. J. Brabec, N. S. Sariciftci, Plastic solar cells, Advanced Functional Materials, vol.11,pg.15-26, 2001.

[6] S. Berkley, The Fabrication and Characterization of Organic Solar Cells, 2009.

[7] M. Filbian, Electronic properties of novel Phthalocyanine based molecular crystals, 2007

[8] G. Turrell and J. Corset, Raman Microscopy, Developments and Applications, Academic Press, London, 1996.

[9] G. Cabailh, I.T. McGovern, Growth of Metal-Phthalocyanine on GaAs (001) an NEXAFS study Proc SPIE 5826, pg. 37-43, 2005.

[10] L. Riele, T. Bruhn, Reconstruction dependent growth of lead phthalocyanine layers on GaAs (001) surfaces, Physical Review B vol. 84, pg. 205317, 2011.

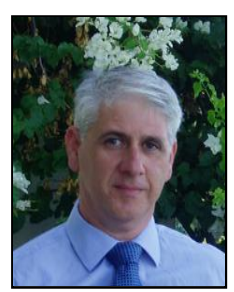

Nikolaos F. Voudoukis received a BSc degree in Physics from Athens National University, Greece, in 1991, a BSc in Electrical and Computer Engineering from the National Technical University of Athens, Greece, in 2012, his MSc degree in Electronics and Telecommunications from Athens National University, in 1993, and his $\mathrm{PhD}$ degree from Athens National University, in 2013. He has worked as telecommunication engineer in Greece. Dr. Voudoukis now is Assistant Director at a high school and a part-time Lecturer at the School of Pedagogical \& Technological. Education, Athens, Greece. 